\title{
Erratum to: Engineering of Microbial Electrodes
}

\author{
Sven Kerzenmacher
}

\section{Erratum to:}

Chapter "Engineering of Microbial Electrodes" in: S. Kerzenmacher, Adv Biochem Eng Biotechnol, DOI: 10.1007/10_2017_16

In 30th page, Table 2, in the last row, the references 33 and 134 are inserted.

\footnotetext{
The updated online version of this chapter can be found at DOI 10.1007/10_2017_16

S. Kerzenmacher $(\bowtie)$

IMTEK - Department of Microsystems Engineering, University of Freiburg, Freiburg im Breisgau, Germany

e-mail: sven.kerzenmacher@imtek.uni-freiburg.de
} 